\title{
Missed opportunities for appropriate diagnosis and management of profound hyponatraemia Audit of District General Hospital experience
}

\author{
P. Cai, C. Thomas, Q. Zeb, C. Anazodo, M. Malik
}

Department of Endocrinology and Diabetes, Scunthorpe General Hospital

\section{Introduction}

Hyponatraemia is a common yet under-investigated metabolic abnormality in the acutely ill patients. This retrospective audit was designed to evaluate the appropriateness of initial biochemical assessment in patients presenting to the Emergency Department of a District General Hospital in the UK.

\section{Objectives}

1. To assess the incidence of profound hyponatraemia among acutely ill patients presenting to the Emergency Department.

2. To ensure that the initial investigation of hyponatraemia is in accordance with the European Society of Endocrinology guidelines.

3. To assess whether recommended investigations were carried out in a timely manner to aid further management.

4. To assess whether the diagnosis of hyponatraemia and management advice was appropriately documented, and communicated to patient's family doctor.

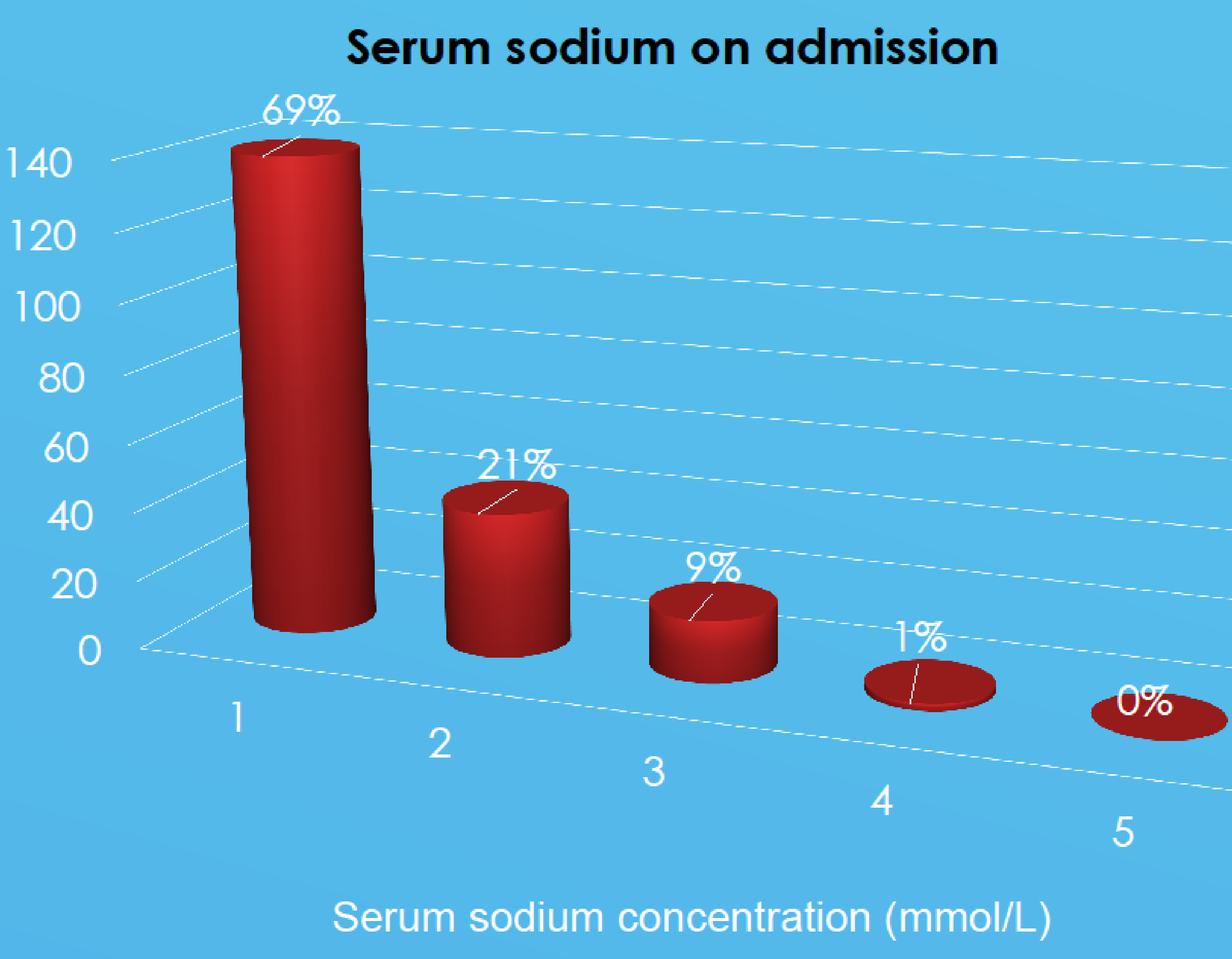

Age distribution

Essential Investigations

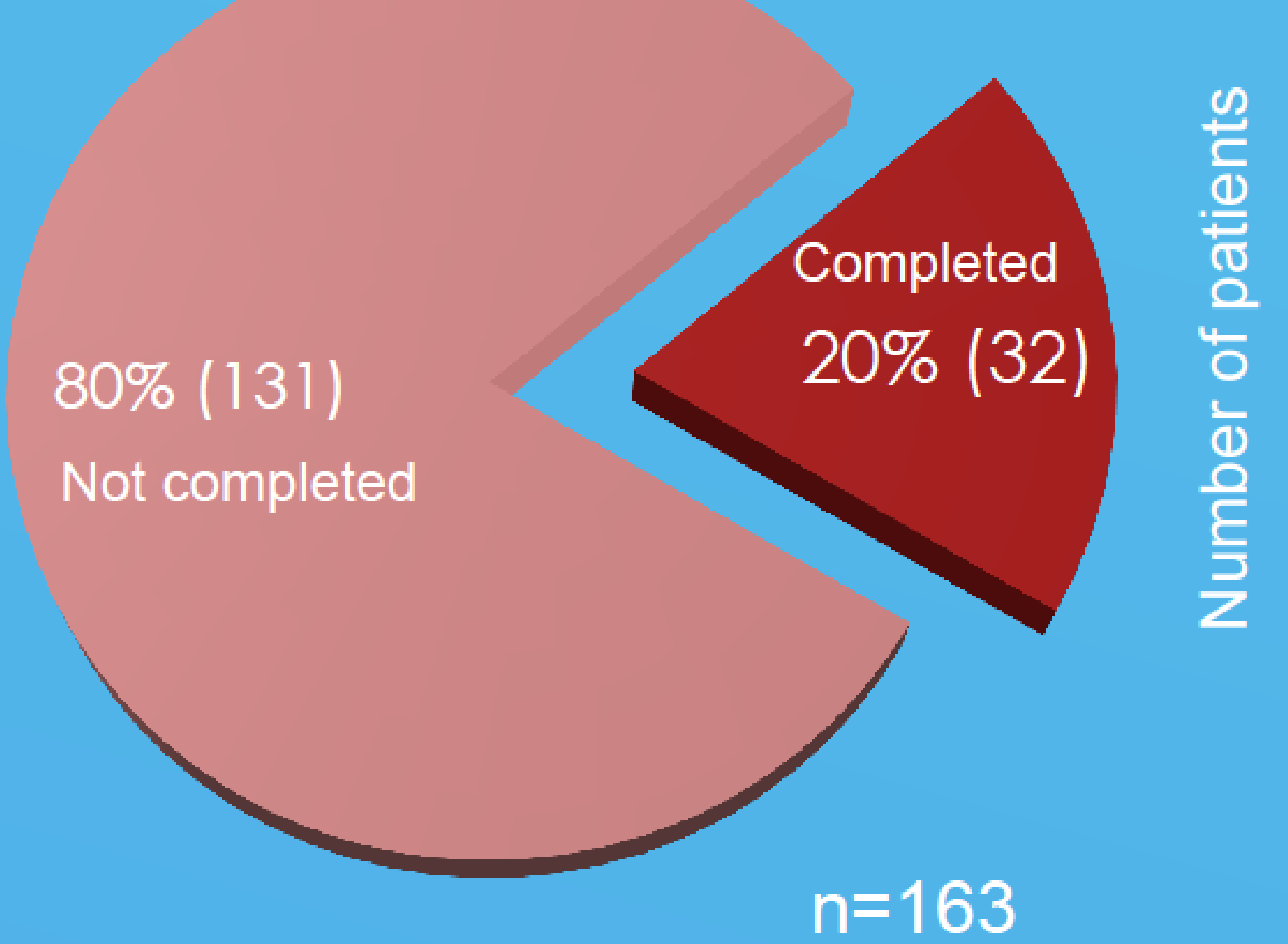

$n=163$

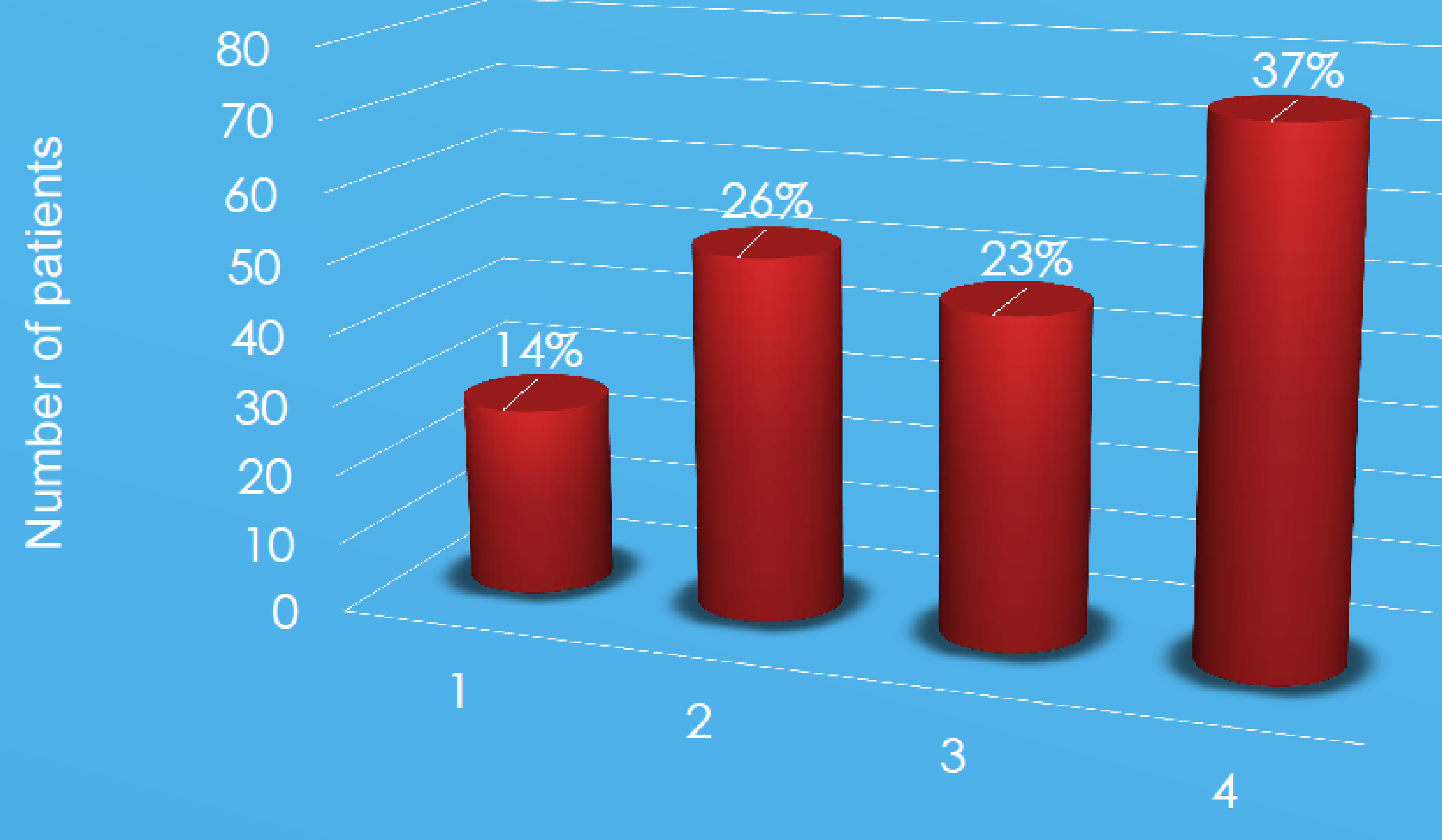

Age range (years)

\section{Methods}

1. Patients admitted via A\&E with profound hyponatraemia (serum $\mathrm{Na} \leq 125 \mathrm{mmol} / \mathrm{L}$ ) from $1^{\text {st }}$ November 2014 to $30^{\text {th }}$ April 2015 were identified using the hospital's laboratory database.

2. Essential and supplementary investigation data were collected from the patients' electronic records on the WebV system.

3. Qualitative data was also collected from the patients' electronic records on the WebV system.

\section{Results}

- $\mathrm{N}=201$ patients (59\% females) with serum sodium of $\leq 125$ were admitted during the six months audit period.

- Serum sodium ranged between 125 and $105 \mathrm{mmol} / \mathrm{L}$, with $10 \%$ of sample $\mathrm{Na}<114$.

- More common in older age ( $37 \%$ of sample was $>80$ years).

- Overall incidence of profound hyponatraemia in patients presenting to A\&E for the audit period was $2.51 \%$.

- In hospital mortality including 6 weeks post-discharge was 19\% (38 patients).

- When deceased patients were excluded, only 32 out of remaining 163 patients (20\%) had complete initial investigations. Of those only 9 patients $(5 \%)$ had these investigations within a timeframe ( $<24$ hours) that can affect further management.

- Discharge Na ranged between 120 to 146 with trend of improvement (average $\Delta+7.87 \%$ ).

- For deceased patients Na level ranged between 113 to 125 , with varied registered cause of death.

- Diagnosis of Hyponatraemia was documented on only 40 (31\%) of patient discharge summaries, and further management advice was given in $34 \%$ of cases.

\section{Conclusions}

Timeframe of essential investigations completed
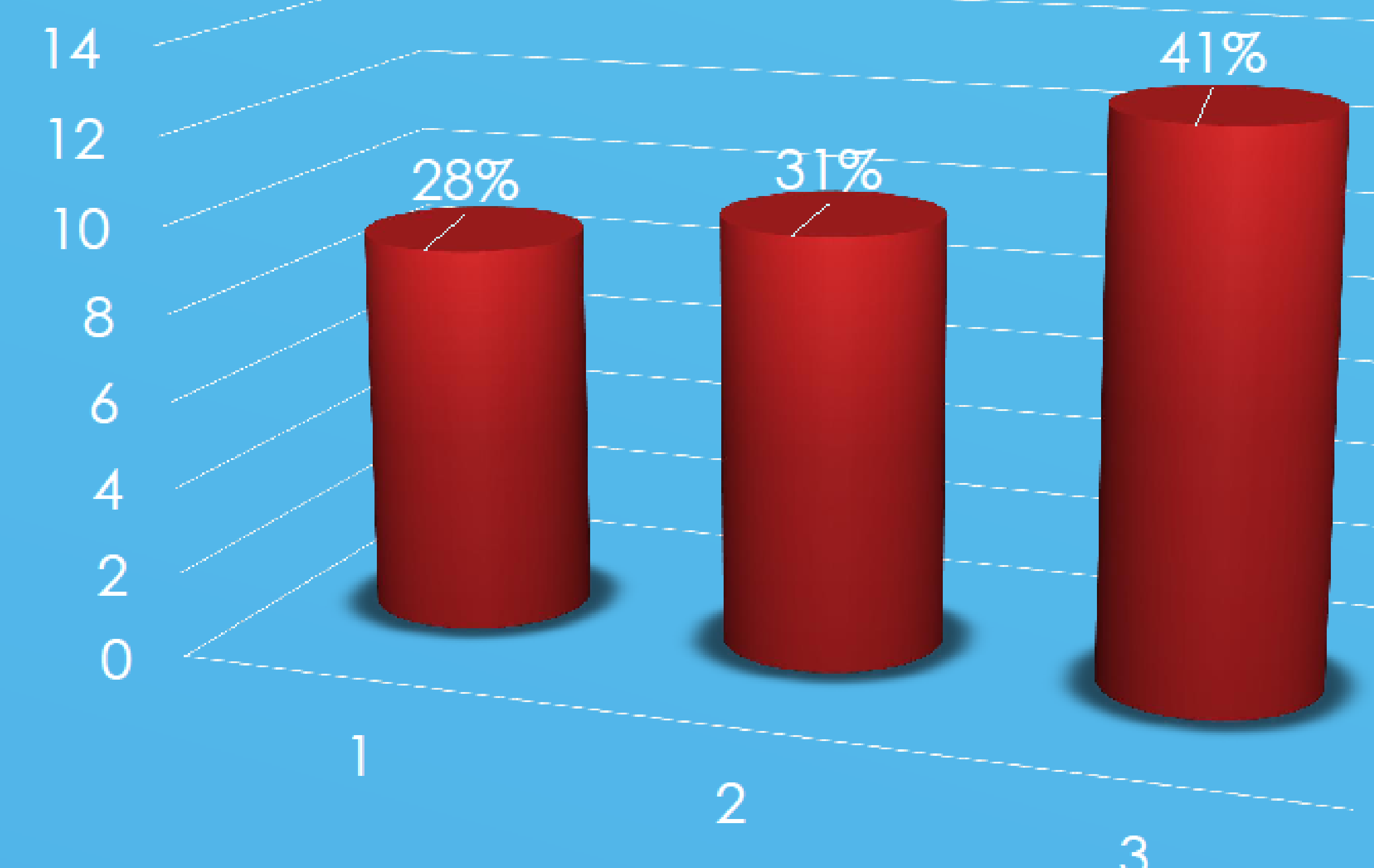

Time (hours)

Significant profound hyponatraemia is common in acutely ill patients, with increased incidence in elderly, and high associated crude mortality. Current practice lags well behind the desired standard of appropriate investigation and management. Four strategies were proposed to improve management; 1) Electronic pop up alert, 2) Readily available checklist, 3) THINK SALT posters at Emergency Department, and 4) Incorporation of diagnosis in patients' records.

\section{References:}

Clinical practice guideline on diagnosis and treatment of hyponatraemia, Spasovski et al, European Journal of Endocrinology (2014) 170, G1-G47 